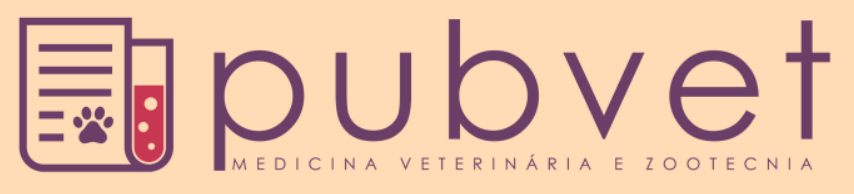

https://doi.org/10.22256/pubvet.v12n3a43.1-5

\title{
Doença de Lyme em cão da raça Rottweiler: Relato de caso
}

\author{
Ana Beatriz Américo Pereira ${ }^{\ominus 1 *}$, Brenda Fernanda Sodré Moreno ${ }^{\circ}$, Lygia Silva \\ Galeno ${ }^{\ominus 2}$, Andressa Mendes Alves ${ }^{\ominus 2}$, Walkyria Conceição Fonseca ${ }^{\ominus 2}$, Sávio Matheus \\ Reis de Carvalho ${ }^{\ominus 3}$, Karoline Gonçalves Moraes ${ }^{\oplus}$, Ana Clara Bastos Rodrigues ${ }^{\bullet}$, \\ Victor Hugo Azevedo Carvalho 06
}

\footnotetext{
${ }^{1}$ Mestranda em Ciência Animal, Universidade Estadual do Maranhão, Campus Paulo VI. São Luís-MA. Brasil.

${ }^{2}$ Discente em Universidade Estadual do Maranhão, Campus Paulo VI. São Luís-MA. Brasil. E-mail: brendafernandasm@gmail.com lygiagaleno@outlook.com, andressamalves@outlook.com,walkyriaconceicao@hotmail.com

${ }^{3}$ Discente em Universidade Federal do Piauí, Campus Profa. Cinobelina Elvas. Bom Jesus - PI. Brasil. E-mail: savioreis1995@ gmail.com

${ }^{4}$ Mestre em Ciência Animal, Universidade Estadual do Maranhão, Campus Paulo VI. São Luís-MA. Brasil. E-mail: karol gm@hotmail.com

${ }^{5}$ Mestranda em Anatomia dos Animais domésticos e Silvestres, Universidade Estadual de São Paulo. São Paulo-SP. Brasil. E-mail: anaclara.rodriguees@hotmail.com

${ }^{6}$ Médico Veterinário autônomo, São Luís-MA. Brasil.E-mail: victorhugo_slip@ hotmail.com

*Autor para correspondência, E-mail: biaap@hotmail.com
}

\begin{abstract}
RESUMO. A doença de Lyme é uma zoonose pouco relatada no Brasil e o cão comportase como reservatório no ambiente domiciliar, o que favorece a transmissão do patógeno para o homem e outros animais. Assim, o objetivo deste trabalho foi relatar a presença de anticorpos anti-Borrelia burgdorferi em um cão atendido em uma clínica de São Luís MA. A doença de Lyme ou borreliose de Lyme é uma enfermidade infecciosa de distribuição mundial, determinada por espiroquetas do gênero Borrelia, que acomete diferentes espécies de animais domésticos, silvestres e também o homem. Foi atendido em uma clínica particular, um cão da raça Rottweiler, macho, 1 ano de idade com a queixa de apatia, secreção ocular bilateral e presença de ectoparasitas. Ao exame físico foi observado que o animal estava magro, mucosas conjuntival e oral hipocoradas, linfonodos submandibular, pré-escapulares e poplíteos normais à palpação e sem alterações à auscultação pulmonar e cardíaca. O presente caso permitiu concluir que as alterações clínicas e hematológicas podem ser inespecíficas, no entanto em regiões onde a incidência de carrapatos é de grande relevância e por ser uma zoonose, torna-se importante a investigação de borreliose em animais que apresentem histórico de carrapato. $\mathrm{O}$ diagnóstico preciso de borreliose canina deve ser feito a fim de instituir a terapêutica adequada para o caso. É de suma importância o controle dos vetores, pois são eles os responsáveis pela transmissão da borreliose. Sendo assim, é necessário que haja orientação do Médico Veterinário para os proprietários de animais infectados minimizando os riscos à saúde humana e animal.
\end{abstract}

Palavras chave: Borreliose, carrapatos, zoonose

\section{Lyme disease in Rottweiler dog: Case report}

ABSTRACT. Lyme disease is a zoonosis that is rarely reported in Brazil and the dog behaves as a reservoir in the home environment, which favors the transmission of the pathogen to humans and other animals. Thus, the objective of this study was to report the presence of anti-Borrelia burgdorferi antibodies in a dog attended at a clinic in São Luís MA. Lyme disease or Lyme borreliosis is an infectious disease of worldwide distribution, determined by spirochetes of the genus Borrelia, which affects different species of domestic animals, wild and also man. It was attended in a private clinic, a dog of the breed Rottweiler, male, 01 year old with the complaint of apathy, bilateral ocular secretion and 
presence of ectoparasites. Physical examination showed that the animal was thin, conjunctival and oral mucosae hypocorate, submandibular, pre-scapular and popliteal lymph nodes normal to palpation and without alterations to pulmonary and cardiac auscultation. The present case allowed to conclude that the clinical and hematological alterations may be non-specific, however in regions where the incidence of ticks is of great relevance and because it is a zoonosis, it becomes important to investigate borreliosis in animals with a history of ticks. The precise diagnosis of canine borreliosis should be made in order to institute the appropriate therapy for the case. Control of vectors is of paramount importance, since they are responsible for the transmission of borreliosis. Therefore, it is necessary that the Veterinarian's guidance is given to the owners of infected animals, minimizing the risks to human and animal health.

Keywords: Borreliosis, Ticks, Zoonoses

\section{Enfermedad de Lyme en perro de la raza Rottweiler: Reporte de un caso}

RESUMEN. La enfermedad de Lyme es una zoonosis poco reportada en Brasil y el perro se comporta como reservorio en el ambiente domiciliar, lo que favorece la transmisión del patógeno para el hombre y otros animales. Así, el objetivo de este trabajo fue relatar la presencia de anticuerpos anti-Borrelia burgdorferi en un perro atendido en una clínica de São Luís - MA. La enfermedad de Lyme o borreliosis de Lyme es una enfermedad infecciosa de distribución mundial, determinada por espiroquetas del género Borrelia, que afecta a diferentes especies de animales domésticos, silvestres y también al hombre. En una clínica particular, un perro de la raza Rottweiler, macho, 1 año de edad con la queja de apatía, secreción ocular bilateral y presencia de ectoparásitos. En el examen físico se observó que el animal estaba flaco, mucosas conjuntival y oral pálidas, ganglios linfáticos submandibulares, pre-escapular y poplíteos normales a la palpación y sin alteraciones a la auscultación pulmonar y cardíaca. El presente caso permitió concluir que las alteraciones clínicas y hematológicas pueden ser inespecíficas, pero en regiones donde la incidencia de garrapatas es de gran relevancia y por ser una zoonosis, es importante la investigación de borreliosis en animales que presenten historial de garrapata. El diagnóstico preciso de la borreliosis canina debe hacerse a fin de establecer el tratamiento adecuado para el caso. Es de suma importancia el control de los vectores, pues son ellos los responsables de la transmisión de la borreliosis. Por lo tanto, es necesario que haya orientación del Médico Veterinario para los propietarios de animales infectados minimizando los riesgos para la salud humana y animal.

Palabras clave: Borreliosis, garrapatas, zoonosis

\section{Introdução}

A doença de Lyme ou borreliose de Lyme é uma enfermidade infecciosa de distribuição mundial, causada por espiroquetas do gênero Borrelia, que acomete diferentes espécies de animais domésticos, silvestres e também o homem (Bennett, 1995). As borrélias geralmente ligadas a infecções em humanos e cães são eubactérias, de forma espiralada, Gram-negativas e microaerófilas; pertencentes à ordem Spirochetales, Família Spirochaetaceae, gênero Borrelia (Wilske et al., 1988).

A transmissão das borrélias pelos carrapatos da família ixodídae pode ocorrer de forma transovariana e/ou transestadial (Randolph et al., 1996). Nos EUA é considerada uma doença comum em humanos (Sheets et al., 2000). No Brasil encontramos uma variação na sorologia para borreliose em cães, de positividade de zero (Dagnone et al., 2002, Labarthe et al., 2003) até 48,2\% (Alves et al., 2014).

Após a exposição ao agente a doença clínica ocorre de 2 a 5 meses, sendo a gravidade e predisposição para o desenvolvimento dos sinais clínicos inversamente proporcionais à idade do animal e ao estado imunológico (Greene, 1998). Os cães são importantes reservatórios de Borrelia burgdorferi, podendo ser isoladas espiroquetas de animais clinicamente sadios (Burgess, 1986). $\mathrm{Na}$ 
espécie canina, a doença se apresenta na maioria das vezes de forma assintomática (Littman, 2004, Littman et al., 2006); porém, quando os animais apresentam sinais clínicos, pode haver comprometimento das articulações, febre, anorexia, letargia, inapetência e dor articular (Dambach et al., 1997, Levy \& Duray, 1988). Nos humanos expostos a Borrelia burgdorferi cerca de 90\% apresentam sinais de doença (Littman, 2004, Littman et al., 2006).

A doença de Lyme é uma zoonose pouco relatada no Brasil e o cão comporta-se como reservatório no ambiente domiciliar, o que favorece a transmissão do patógeno para o homem e outros animais. Assim, o objetivo deste trabalho foi relatar a presença de anticorpos anti-Borrelia burgdorferi em um cão atendido em uma clínica na cidade de São Luís - MA.

\section{Descrição do caso}

Foi atendido em uma clínica particular da cidade de São Luís-MA, um cão da raça Rottweiler, macho, um ano de idade com a queixa de apatia, secreção ocular bilateral e presença de ectoparasitas. Ao exame físico foi observado que o animal estava magro, mucosas conjuntival e oral hipocoradas, linfonodos submandibular, préescapulares e poplíteos normais à palpação e sem alterações à auscultação pulmonar e cardíaca. Foram realizados hemograma e perfil bioquímico (ureia, creatinina, TGO e TGP) pelo analisador hematológico Idexx, onde foi observada anemia normocítica normocrômica, com hematócrito $32 \%$ $(37,3 \%-61,7 \%)$ e trombocitopenia com valores de $94 \mathrm{~K} / \mu \mathrm{L} \quad(148 \mathrm{~K} / \mu \mathrm{L}-484 \mathrm{~K} / \mu \mathrm{L}) . \quad$ Os valores bioquímicos estavam dentro do limite e foi realizado o teste SNAP 4DX, onde foi observada múltipla infecção por erliquiose (E. canis), anaplasmose (A. phagocytophilum) e doença de Lyme (Lyme), foi realizado ainda pesquisa de babesiose, sendo negativo na pesquisa direta. $\mathrm{O}$ animal ficou internado sendo administrado doxiciclina (na dose de $5 \mathrm{mg} / \mathrm{kg}$, BID), fluidoterapia e complexo B, por se tratar de um animal que apresentava positividade para uma zoonose (doença de Lyme) transmitida por vetor artrópode, foi administrado também medicação para controle dos ectoparasitas que havia no animal. Quando apresentou melhora clínica do quadro, recebeu alta com a continuação do tratamento (doxiciclina e complexo B). Após 21 dias de tratamento foi realizada uma nova avaliação hematológica do animal, onde se observou a normalização dos resultados, constatando a eficácia no tratamento.

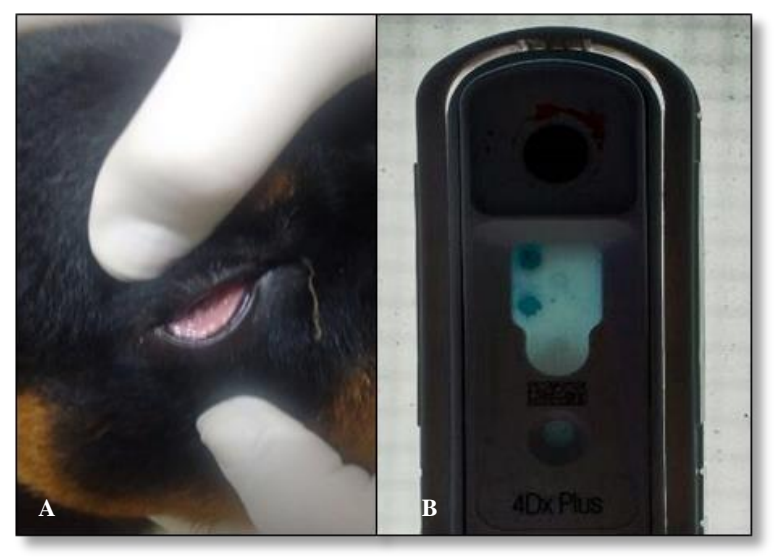

Figura 1. A Mucosa ocular hipocorada. B - Teste positivo para doença de Lyme.

\section{Discussão}

O presente trabalho demonstrou múltipla infecção por erliquiose, anaplasmose e borreliose, pelo kit comercial $\left(\right.$ Snap $4 \mathrm{Dx}^{\circledR}$ ) que testa a presença de anticorpos anti-Anaplasma phagocytophilum, Borrelia borgdorferi, Ehrlichia canis e Dirofilaria immitis. O surgimento das doenças está relacionado com o histórico da presença de carrapato no animal e os sinais clínicos apresentados pelo animal se mostraram inespecíficos de borreliose de Lyme.

Em cães, a associação de hematozoários é frequentemente observada, podendo encontrar-se concomitante Babesia canis, E. canis, Hepatozoon canis e Haemobartonella canis (Iqbal et al., 1994). Têm-se relatado ainda associações entre $E$. canis e E. ewingii e/ou E. platys, e entre Ehrlichia sp., Babesia sp. e B. burgdorferi (Breitschwerdt et al., 1994, Rikihisa et al., 1994). Estas relações entre hematozoários são constantes, pois muitas delas possuem o mesmo carrapato vetor, para cada região (Iqbal et al., 1994, Eremeeva et al., 1995). $\mathrm{Na}$ hematologia, a observação de anemia e trombocitopenia, como ocorreu neste caso, são amplamente citadas, em casos de erliquiose (Kuehn \& Gaunt, 1985). Além disso, Soares et al. (2000) relataram que um quadro de anemia progressiva pode se instalar devido à característica hemolizante da borreliose.

De acordo com Crivellentin \& BorinCrivelletin (2015) nos animais com Doença de Lyme é comum evidenciar apatia, anorexia, febre, linfadenomegalia e claudicação. A poliartrite é a manifestação mais frequente, mas também pode haver dermatite, miosite e glomerulonefrite. A 
terapêutica instituída para o animal está de acordo com o tratamento recomendado pela literatura por antibióticos. Sendo as tetraciclinas, penicilina, ampicilina e amoxicilina as mais efetivas por possuírem melhor absorção, mas a doxicilina é a mais indicada devido à sua característica lipoproteica, que confere maior penetração no tecido (Appel, 1990, Levy \& Dreesen, 1992).

Após o término do tratamento, repetiu-se o hemograma e observou-se que as séries antes alteradas se apresentaram dentro dos valores de referência para a espécie canina.

\section{Conclusão}

O presente caso permitiu concluir que as alterações clínicas e hematológicas podem ser inespecíficas. No entanto em regiões onde a incidência de carrapatos é de grande relevância e por ser uma zoonose, torna-se importante a investigação de borreliose em animais que apresentem histórico de carrapato.

O diagnóstico preciso de borreliose canina deve ser feito a fim de instituir a terapêutica adequada para o caso. É de suma importância o controle dos vetores, pois são eles os responsáveis pela transmissão da borreliose. Sendo assim, é necessário que haja orientação do Médico Veterinário para os proprietários de animais infectados minimizando os riscos à saúde humana e animal.

\section{Referências Bibliográficas}

Alves, A. L., Madureira, R. C., Silva, R. A., Nascimento Corrêa, F. \& Botteon, R. C. M. 2014. Frequiência de anticorpos contra Borrelia burgdorferi em cães na região metropolitana do Rio de Janeiro. Pesquisa Veterinária Brasileira, 24, 203-206.

Appel, M. J. G. 1990. Lyme disease in dogs and cats. Compendium on continuing education for the practicing veterinarian, 12, 617-626.

Bennett, C. E. 1995. Ticks and Lyme disease. Advances in Parasitology, 36, 343-405.

Breitschwerdt, E. B., Nicholson, W. L., Kiehl, A. R., Steers, C., Meuten, D. J. \& Levine, J. F. 1994. Natural infections with Borrelia spirochetes in two dogs from Florida. Journal of Clinical Microbiology, 32, 352-357.

Burgess, E. C. 1986. Natural exposure of Wisconsin dogs to the Lyme disease spirochete (Borrelia burgdorferi). Laboratory Animal Science, 36, 288-290.
Crivellentin, L. Z. \& Borin-Crivelletin, S. 2015. Casos de rotina em medicina veterinária de pequenos animais, São Paulo.

Dagnone, A. S., Trapp, S. M., Jojima, F. S., Amude, A. M., Morais, H. A. S., Freire, R. L. \& Vidotto, O. 2002. Avaliação Soroepidemiológica da infecção por Ehrlichia canis, Dirofilaria immitis e Borrelia burgdorferi em cães de uma população hospitalar. XII Congresso Brasileiro de Parasitologia Veterinária, Rio de Janeiro.

Dambach, D. M., Smith, C. A., Lewis, R. M. \& Van Winkle, T. J. 1997. Morphologic, immunohistochemical, and ultrastructural characterization of a distinctive renal lesion in dogs putatively associated with Borrelia burgdorferi infection: 49 cases (1987-1992). Veterinary Pathology, 34, 85-96.

Eremeeva, M., Balayeva, N., Roux, V., Ignatovich, V., Kotsinjan, M. \& Raoult, D. 1995. Genomic and proteinic characterization of strain S, a rickettsia isolated from Rhipicephalus sanguineus ticks in Armenia. Journal of Clinical Microbiology, 33, 27382744.

Greene, C. E. 1998. Canine distemper. In: Greene, C. E. (ed.) Infections diseases of the dog and the cat. Editora Interamericana, São Paulo.

Iqbal, Z., Chaichanasiriwithaya, W. \& Rikihisa, Y. 1994. Comparison of PCR with other tests for early diagnosis of canine ehrlichiosis. Journal of Clinical Microbiology, 32, 16581662.

Kuehn, N. F. \& Gaunt, S. D. 1985. Clinical and hematologic findings in canine ehrlichiosis. Journal of the American Veterinary Medical Association, 186, 355-358.

Labarthe, N., Campos, P. M., Barbarini, O., McKee, W., Coimbra, C. A. \& Hoskins, J. 2003. Serologic prevalence of Dirofilaria immitis, Ehrlichia canis, and Borrelia burgdorferi infections in Brazil. Veterinary Therapeutics: Research in Applied Veterinary Medicine, 4, 67-75.

Levy, S. A. \& Dreesen, D. W. 1992. Lyme borreliosis in dogs. Canine Practice, 17, 5-14.

Levy, S. A. \& Duray, P. H. 1988. Complete heart block in a dog seropositive for Borrelia burgdorferi: similarity to human Lyme carditis. Journal of Veterinary Internal Medicine, 2, 138-144. 
Littman, M. P. 2004. Lyme disease in dogs. Standards of care. Emergency and Critical Care Medicine, 6, 1-6.

Littman, M. P., Goldstein, R. E., Labato, M. A., Lappin, M. R. \& Moore, G. E. 2006. ACVIM small animal consensus statement on Lyme disease in dogs: diagnosis, treatment, and prevention. Journal of Veterinary Internal Medicine, 20, 422-434.

Randolph, S. E., Gern, L. \& Nuttall, P. A. 1996. Co-feeding ticks: epidemiological significance for tick-borne pathogen transmission. Parasitology Today, 12, 472-479.

Rikihisa, Y., Ewing, S. A. \& Fox, J. C. 1994. Western immunoblot analysis of Ehrlichia chaffeensis, E. canis, or E. ewingii infections in dogs and humans. Journal of Clinical Microbiology, 32, 2107-2112.

Sheets, J. T., Rossi, C. A., Kearney, B. J. \& Moore, G. E. 2000. Evaluation of a commercial enzyme-linked immunosorbent assay for detection of Borrelia burgdorferi exposure in dogs. Journal of the American Veterinary Medical Association, 216, 1418-1422.

Soares, C. O., Ishikawa, M. M., Fonseca, A. H. \& Yoshinari, N. H. 2000. Borrelioses, agents and vectors: a review. Pesquisa Veterinária Brasileira, 20, 1-19.

Wilske, B., Preac-MursicC, V., Schierz, G., Kühbeck, R., Barbour, A. G. \& Kramer, M. 1988. Antigenic variability of Borrelia burgdorferi. Annals of the New York Academy of Sciences, 539, 126-143.

\section{Article History:}

Received 23 October 2017

Accepted 14 November 2017

Available online 11 January 2018

License information: This is an open-access article distributed under the terms of the Creative Commons Attribution License 4.0, which permits unrestricted use, distribution, and reproduction in any medium, provided the original work is properly cited. 